\title{
The Effect of Exercise Order on Maximum Strength Development in Resistance Trainings
}

\author{
Serhat ÖZBAY ${ }^{1}$, Süleyman ULUPINAR², Vedat ÇINAR², Taner AKBULUT ${ }^{3}$
}

${ }^{1}$ Erzurum Technical University, Sport Sciences Fakulty, Erzurum, Turkey

${ }^{2}$ Ph.D. Candidate-Graduate School of Health Sciences, Hacettepe University, Ankara, Turkey2

${ }^{3}$ Department of Movement and Training Science, Firat University, Elazig, Turkey3

Address Correspondence to S. ÖZBAY. serhat.ozbay@erzurum.edu.tr

\begin{abstract}
The aim of this study is to investigate the effect of exercise order on maximum strength development in resistance trainings. Thirty six male wrestlers (age $=21.6 \pm 2.0$ years; height $=169.8 \pm 8.7 \mathrm{~cm}$; body mass $=76.6 \pm 11.1 \mathrm{~kg}$ ) participated in the study . The participants performed two different resistance training programs for 12 weeks. First group (Gr1) performed the exercises involving large muscle groups at initial. The other group (Gr2) performed the same exercises in the opposite order. For the Gr1, the exercise order was bench press (BP), lat pulldown (LPD), shoulder press (SP), biceps curl (BC), triceps extension (TE). For the Gr2, the exercise order was TE, BC, SP, LPD and BP. Before and after the 12-week training period, 1 repeat maximum (1RM) loads were measured in all exercises. According to the results, 1RM loads of all exercises increased significantly in both groups (5.8-16.7\%). However, 1RM load increase in the BP and LPD exercises were significantly higher in Gr1 compared to Gr2. No significant difference was found between the groups in 1RM load increase of SP, BC and TE exercises. As a result it is recommended that exercises involving large muscle groups should be performed first.
\end{abstract}

Keywords: Upper body exercises, Resistance exercises, 1RM strength

\section{INTRODUCTION}

Resistance trainings are commonly preferred by both recreational and professional athletes $(11,13$, 15). Resistance trainings are considered as a valid method because of the mechanical skills that trained can be tested in the same way $(7,20)$. Resistance training components can be design in different ways according to desired characteristics such as hypertrophy, power, muscular endurance or maximal strength $(1,4)$. For example rest periods, weights, exercise types, the number of repetition or set vary in the researches $(4,10,16)$.

Exercise order is stated one of the many factors affecting the performance in resistance trainings, and some recommendations have been presented $(1,3)$. For instance, it was recommended that the exercise order should be performed from multiple joint exercises to single joint exercises (1). Similarly, it was stated that the exercises involving large muscle groups at the beginning of the training and later the exercises involving small muscle groups should be performed to increase performance $(1,3,12)$.

Many of the studies investigating the effect of exercise order on performance examined acute responses to training $(6,8,19)$. In addition, the researchers investigated mostly on amateur or sedentary individuals $(2,14)$. Therefore, the aim of this study is to investigate the effect of exercise order on 1RM strength development in resistance training programs in well-trained athletes.

\section{MATERIAL AND METHOD}

\section{Participants}

Thirty-six well-trained male athletes competing at the highest national level in the Greco-Roman wrestling style participated in the study voluntarily. These athletes have trained regularly for 5 to 10 years. Participants have applied resistance training each year according to the competition calendar, but they have not applied within 6 months prior to this study. Participants were selected from athletes who didn't have current orthopaedic injury and disease. Participants were given detailed explanation about all processes and their informed consent was obtained. For this research, ethics committee approval was obtained from Ethics Committee of Erzurum Atatürk University, Institute of Winter Sports. 


\section{RM test}

$1 \mathrm{RM}$ loads were determined on two different days with 3 days intervals after performing one familiarise training. First day anthropometric characteristics were measured and 1RM loads were determined for barbell bench press (BP), machine lat pulldown (LPD), seated machine shoulder press (SP), free weight seated biceps curl with a straight bar (BC) and machine triceps extension (TE). The same tests were repeated on the second day to ensure test-retest reliability. The greater load between the two test days was considered the valid 1RM load. For each exercise, participants were given three trial with five minutes interval. The other exercise was performed after at least 10 minutes of rest. At the end of the training period, 1RM loads were determined again with the same procedure to examine the change. Test-retest reliability was controlled by intra-class correlation coefficient (ICC). ICC (CI 95\%) values in all exercises were determined between 0.94 (0.91-0.96) - 0.97 (0.94-0.99) before training period, between 0.95 (0.93-0.98) - 0.97 (0.96-0.99) after training period.

\section{Resistance Training}

The participants applied two training programs which included the same exercises in different order. Gr1 performed the exercises ordered from large muscle groups to the small muscle groups while Gr2 performed the same exercises in opposite order. Both groups trained three days a week for 12 weeks on non-consecutive days. The exercise order for Gr1 was BP, LPD, SP, BC and TE. The exercise order for Gr2 was TE, BC, SP, LPD and BP. All exercises were performed in 3 sets for both groups. All trainings were monitored by at least one researcher. Threeminute rest intervals were used between exercises and sets. The subjects tried to reach the maximum number of repetitions at $70 \%$ of $1 \mathrm{RM}$ loads while performing the exercises. If the number of repetitions was among 8-12 participants continued the same load; if less than 8 the load was reduced; if more than 12 the load was increased. The subjects performed a warming consist of 15 repetitions at $50 \%$ of the load to be applied before the first exercise. Attendance rate of participants in training sessions were recorded: $98.1 \%$ for $\mathrm{Gr} 1$; $97.7 \%$ for Gr2.

\section{Statistical Analysis}

All statistical procedures were completed in SPSS 21.0 program. Intra-class correlation coefficient (ICC) was used to ensure reliability in 1RM tests. Shapiro-Wilk test was used to control normality, and Levene's test was used to control homogeneity of variances. The independent sample t-test was used to determine differences between the groups in terms of initial values and in the amount of increase in $1 \mathrm{RM}$ loads. The paired sample t-test was used to determine the differences in the $1 \mathrm{RM}$ loads between before and after training period. The effect size was calculated to determine the practical importance in cases which there was the significant difference statistically (0.2: small; 0.5: medium; 0.8: high). In all statistical procedures $\mathrm{p} \leq 0.05$ was accepted.

\section{RESULT}

The descriptive characteristics and 1RM loads were examined (Table 1), and no significant differences were found between the groups in any of the variables.

Table 1. Descriptive characteristics and 1RM loads before training period $(\mathrm{N}=36)$

\begin{tabular}{lcccc}
\hline & Gr1 $(\mathrm{n}=18)$ & $\mathrm{Gr} 2(\mathrm{n}=18)$ & $\mathrm{t}$ & $\mathrm{p}$ \\
\hline Age (years) & $21.0 \pm 1.8$ & $22.1 \pm 2.2$ & 1.668 & 0.104 \\
\hline Height $(\mathrm{cm})$ & $170.6 \pm 9.6$ & $168.9 \pm 7.9$ & 0.569 & 0.573 \\
\hline BM $(\mathrm{kg})$ & $78.7 \pm 13.4$ & $74.4 \pm 8.6$ & 1.153 & 0.258 \\
\hline BP $(\mathrm{kg})$ & $91.3 \pm 18.8$ & $88.3 \pm 9.6$ & 0.586 & 0.563 \\
\hline LPD $(\mathrm{kg})$ & $87.5 \pm 22.5$ & $85.4 \pm 12.9$ & 0.341 & 0.735 \\
\hline $\mathrm{SP}(\mathrm{kg})$ & $71.1 \pm 13.0$ & $65.1 \pm 9.5$ & 1.576 & 0.124 \\
\hline BC $(\mathrm{kg})$ & $35.8 \pm 8.0$ & $35.1 \pm 8.1$ & 0.260 & 0.796 \\
\hline TE $(\mathrm{kg})$ & $56.7 \pm 14.2$ & $50.3 \pm 10.9$ & 1.514 & 0.139 \\
\hline
\end{tabular}

Table 2. 1RM loads of Gr1 before and after the training period $(\mathrm{n}=18)$

\begin{tabular}{lccccc}
\hline & Before & After & $t$ & $p$ & E.S. \\
\hline $\mathrm{BP}(\mathrm{kg})$ & $91.3 \pm 18.8$ & $105.1 \pm 18.1$ & -27.549 & 0.0001 & 0.75 \\
\hline $\begin{array}{l}\mathrm{LPD} \\
(\mathrm{kg})\end{array}$ & $87.5 \pm 22.5$ & $100.3 \pm 26.1$ & -12.425 & 0.0001 & 0.54 \\
\hline $\mathrm{SP}(\mathrm{kg})$ & $71.1 \pm 12.9$ & $79.4 \pm 14.3$ & -13.038 & 0.0001 & 0.61 \\
\hline $\mathrm{BC}(\mathrm{kg})$ & $35.8 \pm 8.0$ & $41.8 \pm 10.1$ & -8.152 & 0.0001 & 0.66 \\
\hline $\mathrm{TE}(\mathrm{kg})$ & $56.7 \pm 14.2$ & $62.6 \pm 15.8$ & -11.926 & 0.0001 & 0.39 \\
\hline
\end{tabular}

The differences between before and after the training period for Gr1 was examined (Table 2). It was found that 1RM loads of all exercises increased significantly after training period compared to 
before. The results were evaluated based on practical importance and it was found that BP, LPD, $\mathrm{SP}$ and $\mathrm{BC}$ exercises had medium effect size (ES= $0.75 ; 0.54 ; 0.61$ and 0.66 , respectively); TE exercise had low effect size ( $E S=0.39$ ).

Table 3. 1RM loads of Gr2 before and after the training period $(\mathrm{n}=18)$

\begin{tabular}{lccccc}
\hline & Before & After & $\mathrm{t}$ & $\mathrm{p}$ & E.S. \\
\hline $\mathrm{BP}(\mathrm{kg})$ & $88.3 \pm 9.6$ & $93.5 \pm 10.4$ & -16.171 & 0.0001 & 0.52 \\
\hline $\begin{array}{l}\mathrm{LPD} \\
(\mathrm{kg})\end{array}$ & $85.4 \pm 12.8$ & $91.9 \pm 12.6$ & -18.230 & 0.0001 & 0.51 \\
\hline $\mathrm{SP}(\mathrm{kg})$ & $65.1 \pm 9.5$ & $70.4 \pm 10.0$ & -13.701 & 0.0001 & 0.54 \\
\hline $\mathrm{BC}(\mathrm{kg})$ & $35.1 \pm 8.0$ & $40.6 \pm 8.9$ & -17.867 & 0.0001 & 0.65 \\
\hline $\mathrm{TE}(\mathrm{kg})$ & $50.3 \pm 10.9$ & $56.9 \pm 11.4$ & -19.044 & 0.0001 & 0.59 \\
\hline
\end{tabular}

The difference between before and after the training period for Gr2 was examined (Table 3). It was found that $1 \mathrm{RM}$ loads of all exercises increased significantly after training period compared to before. The results were evaluated in terms of practical importance and it was found that all $\mathrm{BP}$, LPD, SP, BC and TE exercises had medium effect size (ES $=0.52 ; 0.51 ; 0.54 ; 0.65$ and 0.59 respectively).

Figure 1. Increases in amount of $1 \mathrm{RM}$ loads of the groups (kg and \%)

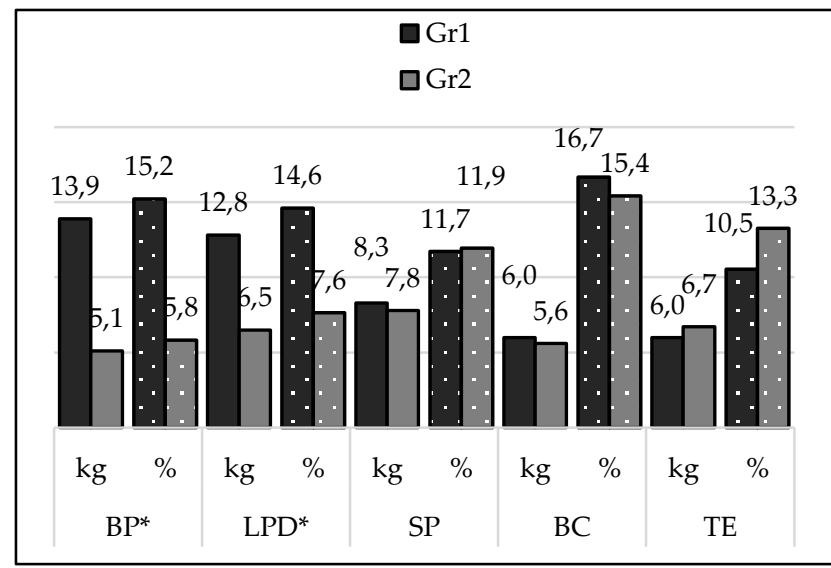

* There is significant difference between the groups in 1RM load increase amount.

Increase amounts in 1RM loads ( $\mathrm{kg}$ and \%) depending on the training period in Gr1 and Gr2 were examined (Figure 1). There was significantly higher 1TM load increase for BP and LPD exercises in Gr1 compared to Gr2. There was no significant difference in 1RM load increase amount of SP, BC and TE exercises between the groups.

\section{DISCUSSION}

In present study, it is aimed to examine the effect of exercise order on 1RM strength performance. It was found that 1RM loads increased significantly in both groups who performed same exercises the opposite order. However, in the group that applied large muscle group exercises first, in BP and LPD exercises there was higher 1RM load increase compared to the other group. In 1RM load increase of SP, BC and TE exercises, there was no significant difference between the groups.

The effect of exercise order on performance was studied in different ways. Kazem et al. (2014) shown that exercise order from upper body to lower body or the opposite had no significant effect on performance (8). Fisher et al. (2014) used chest press, leg press, and pull-down exercises, and they found that the exercise order had no effect on performance (5). However, in our study, unlike designs of these studies, the order of exercise was performed from large muscle groups to small muscle groups or the opposite.

To increase the efficiency of the trainings, it is stated that the exercises involving large muscle groups should be performed first $(1,3,12)$. It is possible to reach different results in the studies. For example, Kazem et al. (2013) found that there was no significant difference between the groups who applied the exercises from the small to the large muscle groups or from the large to the small muscle groups. On the other hand, in two different studies examining the effect of exercise order on rated perceived exertion, Simao et al. (2007) found that exercise order have no effect on perceived exertion rated, whereas Sheikholeslami-Vatani et al., (2016) found that participants assessed as more difficult the training session which performed small muscle groups first $(17,19)$. In another study, it was found that the number of repetitions were increased when exercises were performed first, independent of the exercise order (18).

In another study using the same exercises with our study, it was found that, in BP and LPD exercises, the number of repetitions were higher in the group who performed large muscle groups first, whereas in TE exercise, the number of repetitions were higher in the group who performed the small muscle groups first. There was no significant

difference between the groups in $\mathrm{SP}$ and $\mathrm{BC}$ exercises (9). Dias et al. (2010) found that there was 
significant performance increase in both groups who performed the same exercises in different orders after the eight-week training program. They found that there was greater performance increase in TE exercise in the group that first performed the exercises involving small muscle groups. However, they found that performance increases in BP, LPD, $\mathrm{SP}$, and $\mathrm{BC}$ exercises were similar and there was no significant difference between the groups. In our study, there was higher performance increase in BP and LPD exercises in the group who performed the exercises involving large muscle groups first, whereas SP, BC and TE exercises were not significantly different between the groups. However, in their research, Dias et al. (2010) studied on untrained individuals while our study was conducted on well-trained athletes.

\section{CONCLUSION}

According to the results of our study, it is recommended that exercises involving large muscle groups should be performed first. Because, in cases the exercises involving small muscle groups are performed, local fatigue occurred in small muscle groups may reduce performance during the exercises involving large muscle groups are performed. In other words, excessive fatigue in small muscle groups may cause early ending of exercise, although there is not enough fatigue in large muscle groups. The results of this study involve well-trained athletes and only upper-body exercises. The results of this study involve welltrained athletes and only upper-body exercises. In future studies, more comprehensive results can be found related to the effect of exercise order on performance in different participant groups and lower body exercises.

\section{REFERENCES}

1. ACSM. American College of Sports Medicine position stand. Progression models in resistance training for healthy adults. Medicine and science in sports and exercise, 2009; 41: 687.

2. Buskard A, Wood R, Mullin E, Bruneau M, Jaghab A, and Thompson B. Heart Rate Determined Rest Intervals in Hypertrophy-Type Resistance Training. Journal of Exercise Physiology Online, 2017; 20.

3. Eddens L, van Someren K, and Howatson G. The Role of Intra-Session Exercise Sequence in the Interference Effect: A Systematic Review with Meta-Analysis. Sports Medicine, 2018; $1-12$.

4. Dias I, de Salles BF, Novaes J, Costa PB, and Simão R. Influence of exercise order on maximum strength in untrained young men. Journal of Science and Medicine in Sport, 2010; 13: 65-69.

5. Fisher J, Steele J, and Smith D. High-and low-load resistance training: interpretation and practical application of current research findings. Sports Medicine, 2017; 47: 393-400.
6. Fisher JP, Carlson L, Steele J, and Smith D. The effects of preexhaustion, exercise order, and rest intervals in a full-body resistance training intervention. Applied Physiology, Nutrition, and Metabolism, 2014; 39: 1265-1270.

7. Fleck SJ and Kraemer W. Designing Resistance Training Programs, 4E. Human Kinetics, 2014.

8. Forti LN, Van Roie E, Njemini R, Coudyzer W, Beyer I, Delecluse $\mathrm{C}$, and Bautmans I. Effects of resistance training at different loads on inflammatory markers in young adults. European journal of applied physiology, 2017; 117: 511-519.

9. Kazem S, Aliakbar A, and Bahman M. The comparison of three type of exercise sequence on maximum strength in untrained young men. Физическое воспитание студентов, 2014; 65-69.

10. Kazem S, Bahman M, and Farhad R-N. Influence of upperbody exercise order on muscle damage in untrained men. Physical education of students, 2013; 17: 100-105.

11. Konopack JF. Resistance Training: Recommendations for Age-Relevant Benefits. Annual Review of Gerontology \& Geriatrics, 2016; 36: 193.

12. McKinlay BJ, Wallace $\mathrm{P}$, Dotan R, Long D, Tokuno C, Gabriel D, and Falk B. Effects of Plyometric and Resistance Training on Muscle Strength, Explosiveness and Neuromuscular Function in Young Adolescent Soccer Players. Journal of strength and conditioning research, 2018.

13. Moraes E, Nobre MP, deFreitas Maia M, deFreitas Salles B, Miranda $\mathrm{H}$, and Simão R. Influence of exercise order on the number of repetitions in untrained teenagers. Manual Therapy, Posturology \& Rehabilitation Journal= Revista Manual Therapy, 2016; 14.

14. Murphy PG, Dominguez D, and Karabulut M. One Set to Failure per week Increases Strength More Effectively than Traditional Resistance Training: 1789 Board\# 50 May 312. Medicine \& Science in Sports \& Exercise, 2018; 50: 420.

15. Paoli A, Moro T, Bianco A, Neri M, Battaglia G, and Palma A. Metabolic Effects of Eight Weeks of Intermittent Fasting and Resistance Training in Amateur Body Builders. Medicine \& Science in Sports \& Exercise, 2015; 47: 233.

16. Schoenfeld BJ, Ogborn D, and Krieger JW. Dose-response relationship between weekly resistance training volume and increases in muscle mass: A systematic review and metaanalysis. Journal of sports sciences, 2017; 35: 1073-1082.

17. Schoenfeld BJ, Wilson JM, Lowery RP, and Krieger JW. Muscular adaptations in low-versus high-load resistance training: A meta-analysis. European journal of sport science, 2016; 16: 1-10.

18. Sheikholeslami-Vatani D, Ahmadi S, and Salavati R. Comparison of the effects of resistance exercise orders on number of repetitions, serum IGF-1, testosterone and cortisol levels in normal-weight and obese men. Asian journal of sports medicine, 2016; 7.

19. Simão R, Farinatti PdTV, Polito MD, Maior AS, and Fleck SJ. Influence of exercise order on the number of repetitions performed and perceived exertion during resistance exercises. The Journal of Strength \& Conditioning Research, 2015; 19: 152-156.

20. Simao R, Farinatti PDTV, Polito MD, Viveiros L, and Fleck $\mathrm{SJ}$. Influence of exercise order on the number of repetitions performed and perceived exertion during resistance exercise in women. The Journal of Strength \& Conditioning Research, 2007; 21: 23-28.

21. Sousa D, Piraua A, Beltrao N, JÚNIOR DL, Oliveira L, NETO $\mathrm{AL}$, and Araujo R. Effect of exercise order on multiple onerepetition maximal test performance. MED SPORT, 2016; 69: $1-2$ 